\title{
La Interseccionalidad como Herramienta de Análisis del Fracaso Escolar y del Abandono Educativo: Claves para la Equidad
}

\author{
Intersectionality as a Tool for Analysis of School Failure and \\ Dropping Out: Keys to Equity
}

\author{
Rosa Vázquez * \\ Universidad de Cádiz, España
}

\begin{abstract}
El propósito de este trabajo es analizar el fracaso escolar y abandono educativo desde una perspectiva interseccional. La investigación cualitativa ha sido el posicionamiento metodológico asumido, con las estrategias de recogida de información ad hoc y desde el que ha sido posible afrontar el proceso de análisis. Los resultados permiten identificar la matriz de dominación que se configura a partir de los diversos sistemas de dominación que juegan un papel clave en la concurrencia del fracaso escolar y del abandono educativo. Se abordan los cuatros dominios de los que se compone: estructural, disciplinario, interpersonal y hegemónico. Se concluye con la evidencia de que la interseccionalidad nos permite situarnos en los dispositivos que definen y causan desigualdades e injusticias, o por el contrario, provocan experiencias y situaciones de privilegio. Incorporar la herramienta de la interseccionalidad para el análisis del fracaso escolar y del abandono educativo nos permite reforzar la idea de que no son neutrales, estáticos e inmanentes. La interseccionalidad, como herramienta de análisis crítico, resulta de gran valor para luchar por los derechos de quienes son excluidos, discriminados, marginados desde un proyecto educativo, social y político, comprometido con el bien común y la equidad.
\end{abstract}

Descriptores: Fracaso escolar; Abandono educativo; Interseccionalidad; Justicia social; Equidad.

\begin{abstract}
The purpose of this paper is to analyse school failure and dropping out from an intersectional perspective. The qualitative research has been the methodological positioning assumed, with ad hoc information collection strategies and from which it has been possible to face the analysis process. The results allow to identify the matrix of domination that is configured from the various systems of domination that play a key role in the concurrence of school failure and dropping out. The four domains of which it is composed are addressed: structural, disciplinary, interpersonal and hegemonic. It concludes with the evidence that intersectionality allows us to situate ourselves in the devices that define and cause inequalities and injustices, or on the contrary, provoke experiences and situations of privilege. Incorporating the intersectionality tool for the analysis of school failure and dropping out allows us to reinforce the idea that they are not neutral, static and immanent. Intersectionality, as a tool for critical analysis, it is our ally to fight for the rights of those who are excluded, discriminated against, marginalized with an educative project, social and political, committed to the common good, equity and social justice.
\end{abstract}

Keywords: School failure; Dropping out; Intersectionality; Social justice; Equity.

*Contacto: rmaria.vazquez@uca.es

ISSN: 2254-3139

www.rinace.net/riejs/

revistas.uam.es/riejs
Recibido: $\quad 20$ de febrero 2020

$11^{\text {a }}$ Evaluación: 21 de abril 2020

$2^{\text {a }}$ Evaluación: 1 de junio 2020

Aceptado: 29 de junio 2020 


\section{Introducción}

La presente disertación tiene como propósito analizar, desde una perspectiva interseccional, la producción del fracaso y del abandono educativo. Un abordaje que toma la matriz configurada a partir de los diversos sistemas de dominación que, entendemos, juegan un papel clave en la concurrencia de tales fenómenos, que, si atendemos a su génesis y no solo a su legitimación y cristalización, como señala Rujas (2017), no siempre han sido un problema social y público como lo es en nuestros días. El sistema educativo, pese a sus intentos, encubre el adosamiento de las políticas neoliberales y conservadoras que acarrean desigualdad, inequidad e injusticia.

En este proceso de análisis, la interseccionalidad (Hill Collins, 1990, 2009) se nos presenta como una herramienta adecuada. Aunque los trabajos de Hill Collins nacen en el contexto epistemológico del feminismo negro, su propuesta tiene un punto de partida común con el posicionamiento que adoptamos para el estudio del fracaso escolar y abandono educativo. Respecto a este, se constata la proliferación de estudios e investigaciones que se han desarrollado tanto en el contexto nacional como internacional, constituyendo un punto de referencia importante. Sin embargo, en este amplio conjunto de aportes, las voces y experiencias de los adolescentes que se encuentran en situación de riesgo (at risk) de abandonar o ya lo han hecho no ocupan un lugar central. Este es el punto que conecta con la propuesta de Hill Collins. La autora coloca las experiencias de las mujeres negras estadounidenses en el centro del análisis, sin privilegios, dándoles el lugar y el reconocimiento que le son propios como derecho. Defiende que la comprensión de las desigualdades y la opresión a las que están sometidas las mujeres negras pasa por situar el foco de análisis en sus experiencias en las que entran en juego, de manera interseccional, los diferentes sistemas de desigualdad/dominación. De este modo, el conocimiento que se deriva del análisis de las experiencias es sumamente valioso, en la medida que emerge de una epistemología encarnada en los sujetos que experimentan y sufren exclusión (háblese de las mujeres negras como del alumnado que experimenta fracaso, abandona o está en camino de hacerlo), que ayuda a comprender y explicar el fracaso y el abandono sin desatender a la desigualdad de condiciones y oportunidades a las que se enfrenta el alumnado. Por ello, entendemos que resulta oportuno situar el análisis del constructo ideológico del fracaso y del abandono en esta perspectiva de análisis (Hill Collins, 1990, 2009; Hill Collins y Bilge, 2019).

En lo que sigue, se establecen los aspectos de referencia, teóricos y conceptuales, del fracaso escolar y del abandono educativo, que enlazan con el tratamiento de la interseccionalidad como herramienta de análisis. Gracias a esta, se presenta la matriz de dominación de ambos fenómenos configurada a partir de los resultados de la investigación desarrollada, para concluir con algunas claves que nos ayuden a conseguir una educación basada en la equidad.

\section{La matriz teórica del fracaso escolar y del abandono educativo}

El fracaso escolar y el abandono educativo son dos asuntos que suscitan una gran preocupación y se reconoce como un problema público y social, nacional e internacional, al que hay que dar respuestas. España se sitúa con un porcentaje de un 17,3\% (Ministerio 
de Educación y Formación Profesional, 2020) en abandono educativo temprano. Pese a la progresiva reducción, no constante, que ha experimentado (del 36,4\% en 1994 al 17,3\% en 2019), el porcentaje está aún distanciado de la media europea que se sitúa en el 10,6\%, y también con respecto a los objetivos establecidos por la Comisión Europea para el año 2020, que fija la tasa en un 10\% (Comisión Europea, 2010). España, en el marco de esta estrategia, y desde el 2011, se ha propuesto reducir la tasa de abandono educativo prematuro por debajo del 15\%, como queda recogido en los Programas Nacionales de Reforma del Ministerio de Hacienda ${ }^{1}$.

Unido a los datos que nos ofrecen los organismos nacionales e internacionales, contamos con un corpus amplio de investigaciones y estudios. En este sentido, han sido numerosos los que hasta la fecha se han desarrollado y constituyen un soporte relevante para la explicación y comprensión de dichos fenómenos. El conjunto de trabajos realizados a nivel nacional e internacional aporta factores que intervienen en el abandono educativo. Tomadas en su conjunto y asumiendo las similitudes y las diferencias entre las mismas, estas investigaciones dan cuenta de factores que tienen que ver con el nivel social, cultural y económico de la unidad familiar, el origen social y económico del estudiantado, la inmigración y ser estudiante inmigrante, el género, el rendimiento académico, las características del alumnado, las características de las escuelas, el contexto comunitario y social, etc. (Bernardi y Requena, 2010; Breen y Jonsson, 2005; Calero, 2007; Calero, Choi y Waisgrais, 2010; Entorf y Minoiu, 2005; Escudero, 2005; Escudero, González y Martínez, 2009; Ferrer, Castel y Ferrer, 2006; Finn y Rock, 1997; Marchesi, 2000, 2003; Mora, 2010; Moriña Díez, 2007; Romero y Hernández, 2019; Rumberger y Ah Lin, 2008; Witte et al., 2013). Los factores que intervienen, en mayor o menor grado, dibujan un mapa complejo, si bien los modelos estadísticos que puedan establecerse a partir de aquellos solo pueden sugerir, pero no probar conexiones causales (Rumberger, 2001, p. 9). Este estado de factores necesita de las voces del estudiantado, que es en quien al final se materializa el fracaso y el abandono (Calderón, 2014, 2016a, 2016b; Ferguson et al., 2005; Fernández, Mena y Riviére, 2010; Mena, Fernández y Riviére, 2010; Parrilla et al., 2010; Rumberger, 2001, 2011; Susinos y Calvo, 2005, 2006; Vázquez, 2018).

Atendiendo a los conceptos en sí, y tomando la definición que establece la Comisión Europea, el abandono educativo (school leavers) remite a jóvenes de 18 a 24 años que no están escolarizados y que no han completado la educación secundaria obligatoria o niveles educativos anteriores. Son estudiantes que posee ISCED 0, 1, 2 y 3 (UNESCO, 2012). Por otra parte, el fracaso escolar cuenta con definiciones diversas que muestran su complejidad, las cuales convergen en el alumnado que no logra el título educativo obligatorio y en aquel que no alcanza el nivel de conocimientos mínimos ni el rendimiento académico establecido para la educación obligatoria. No obstante, no podemos olvidar que son constructos sociales teñidos de ideología. Como señala Carabaña (2009),

el fracaso escolar es un arbitrario administrativo que no refleja el aprendizaje de los alumnos y carece de validez y de fiabilidad como indicador de la calidad de la enseñanza. Más aún, en la medida en que tiene consecuencias sociales, su arbitrariedad es una grave injusticia. (p. 76)

\footnotetext{
1 Portal del Ministerio de Hacienda: https://www.hacienda.gob.es/glES/CDI/Paginas/EstrategiaPoliticaFiscal/ProgramaNacionalReformas.aspx
} 


\section{La interseccionalidad: Herramienta para un análisis crítico}

A la luz de los estudios y las investigaciones disponibles, se evidencia la complejidad de los fenómenos que abordamos a través de la trama que se configura a partir de un conjunto diverso y extenso de factores, que remiten a las particularidades del sistema educativo, de las familias y sus contextos, de los centros educativos y las aulas, y del estudiantado en particular. Esta evidencia constata el hecho de que el fracaso escolar y el abandono educativo no son constitutivos del alumnado. Al mismo tiempo, este hecho revela cómo otros sistemas quedan soterrados en el elenco de factores considerados (por ejemplo, el sistema económico y el sistema patriarcal). La comprensión del fracaso escolar y del abandono educativo, que los entendemos como una situación de desigualdad educativa (no al margen de otras posibles desigualdades), se enriquece de forma clara y significativa de las aportaciones que ofrece la interseccionalidad (Crenshaw, 1991; Hill Collins, 1990, 2009; La Barbera, 2016), como herramienta de análisis estructural y como indagación crítica para interpretar las interrelaciones que existen a nivel individual, social e institucional (Hill Collins y Bilge, 2019) en la conformación del fracaso y del abandono educativo. En palabras de las autoras,

[la] interseccionalidad es una forma de entender y analizar la complejidad del
mundo, de las personas y de las experiencias humanas. Los sucesos y las circunstancias
de la vida social y política y la persona raramente se pueden entender como
determinadas por un solo factor. En general están configuradas por muchos factores
y de formas diversas que se influyen mutuamente. En lo que se refiere a la desigualdad
social, la vida de las personas y la organización del poder en una determinada
sociedad se entienden mejor como algo determinado, no por un único eje de la división
social, sea este la raza, el género o la clase, sino por muchos ejes que actúan de manera
conjunta y se influyen entre sí. La interseccionalidad como herramienta analítica
ofrece a las personas un mejor acceso a la complejidad del mundo y de sí mismos. (pp.
$13-14$ )

Las diferentes desigualdades (social, cultural, educativa, política, económica) que sufre el sujeto son ejes que interseccionan momentos y situaciones de la vida del alumnado que experimenta el fracaso y el abandono. La noción de interseccionalidad nos coloca en la interdependencia y en la interacción, en esos puntos de cruces tejidos por las diferentes dimensiones, estructuras y dinámicas que determinan el fracaso escolar y el abandono educativo, y nos sitúa, además, en los dispositivos que definen y causan la desigualdad, la discriminación y la exclusión, o por el contrario, provocan experiencias y situaciones de privilegio.

De esa interseccionalidad emanan diferentes formas de dominación bajo las que el alumnado construye sus identidades (de género, cultural, étnica, de clase social, etc.), que pueden entran en confrontación por efecto de esas formas de dominación. Estas remiten al patriarcado, a la xenofobia, al colonialismo, etc., pero también al propio fracaso. Este se configura como una forma de dominación, de base relacional, que reproduce las desigualdades ya existentes y produce otras, con grados diferentes y con múltiples y diversas maneras de expresión según las realidades de cada sujeto, y con un carácter acumulativo que generan estructuras durables que inyectan discriminación y marginación. El estudiantado perteneciente a las categorías de fracaso escolar y de quienes han abandonado se sitúa en esa intersección, de ahí que resulte necesario enfocar el análisis en los sujetos para poder comprender el modo en que esos factores y sistemas inciden y 
condicionan sus experiencias, las cuales se desarrollan y configuran en contextos sociales y educativos desiguales; contextos que también sufren la desigualdad que generan sobre estos los diferentes sistemas (económico, social, patriarcal, etc.).

La pertenencia a categorías, sean las mencionadas, o cualquier otra que aparta a los otros del nosotros (entendiendo por este grupo aquel que tiene experiencias y situaciones de privilegio y que responde a lo esperado, a la norma), lleva adheridas desigualdades categoriales (Tilly, 2000) que pueden llegar a ser estables y persistentes, y aunque se produzcan cambios que permitan pasar de un grupo a otro (de una categoría a otra), el estigma permanece como dispositivo simbólico del fracaso y/o del abandono. Estas desigualdades categoriales explican, en no pocas ocasiones, la acumulación de oportunidades y disfrute de las mismas por el grupo nosotros, y la escasez que experimentan los otros; una relación de desigualdad que puede tener persistencia en el tiempo, más cuando la desigualdad de resultados de una generación es la desigualdad de oportunidades de la siguiente generación (Giddens, 2000, p. 99). Ese estudiantado (el del grupo de los otros, de los que fracasan, de los que abandonan) termina formando un grupo social -no homogéneo en su seno, en correspondencia con lo que McCall (2005) establece en términos de la complejidad intracategorial- que es excluido, no quedando al margen de esta exclusión la situación socioeconómica, la identidad cultural, la identidad de género ${ }^{2}$, etc. De ahí la importancia, y siguiendo a Tilly (2000), de atender a las diferencias categoriales, ya que "explican realmente gran parte de lo que los observadores comunes y corrientes toman como resultados de la variación en el talento o el esfuerzo individuales" (p.20). Tener este acercamiento heurístico ante los fenómenos de fracaso escolar y abandono educativo es realizable con la herramienta de la interseccionalidad, evitando caer en simplificaciones explicativas que redundan no favorablemente en las medidas de intervención/actuación a adoptar.

Por tanto, no hay un alumnado universal, ni un alumnado que fracasa y/o abandona universal. Consecuentemente, el carácter nomotético atribuible al fracaso y al abandono se debilita para tomar un carácter idiosincrásico contextualizado que mira a las estructuras y a sus dispositivos que conforman una matriz de dominación (Hill Collins, 1999, 2009) como resultado de su intersección. De igual modo, las respuestas educativas no pueden ser generales ni estandarizadas, enmarcadas en programas específicos dirigidos a la población estudiantil que pertenece a la categoría de fracaso escolar o de riesgo de abandono educativo, como se viene haciendo (Escudero y Martínez, 2012; Tarabini y Bonal, 2016), pues, aunque haya un intento de resolver, se obtiene el reforzamiento de las desigualdades. La herramienta de la interseccionalidad, desde su valor crítico y carácter práctico, nos ayuda a detectar y analizar las dimensiones tangibles y las que menos para evitar que los efectos de los sistemas que generan desigualdad, inequidad e injusticia entren a formar parte del imaginario social y educativo como si fuera algo inevitable.

Asimismo, y pese a los discursos inclusivos de las políticas educativas actuales, se persigue un alumnado que se ajuste a las exigencias que marcan los mercados en el escenario de la sociedad neoliberal de capitalismo financiero, y que reclaman el conocimiento que organismos como la OCDE, el FMI, el BM y la OMC necesitan para continuar asegurando y fortaleciendo su poder económico, político y social. Dichas exigencias calan en las determinaciones políticas y educativas, y en las estructuras organizativas, reconfigurando

${ }^{2}$ Véase, por ejemplo, el trabajo de Calvo García (2018). 
la educación. Desde esos organismos se presiona a los sistemas educativos para introducir cambios en estos para así poder aumentar las oportunidades y los beneficios de la economía imperante (Williamson, 2019). Así, el alumnado no solo debe responder a los estándares establecidos, sino también a la norma estandariza de lo que es ser un buen alumno para una institución, que es tomada como instrumento para canalizar las medidas que responden a la ideología economicista y empresarial que promueven los organismos y los grupos económicos y políticos más poderos del momento. Cualquier desvío de la norma coloca al sujeto en una situación de exclusión y desigualdad que, tarde o temprano, traerá consigo prejuicios, estigmas, etiquetas que reforzarán dicha exclusión y desigualdad. Hablar de estándares y de estudiantes estándar, cuando se analizan los fenómenos de fracaso escolar y abandono educativo, obviando los efectos de tales prejuicios, vinculados a cuestiones estructurales, es un modo de eliminar a todos aquellos estudiantes no normativizados -sujetos desviados que se aparta del colectivo estándar y homogéneo requerido y que pertenecen, a su vez, a la categoría de los otros-, por un lado, y de borrar toda posibilidad de cuestionar las prácticas no inclusivas que no dan respuestas a las necesidades reales de esos estudiantes, por otro. Tal vez tengamos que empezar a poner en el epicentro del análisis, como hicieron Alonso y otros (2009), la educación que recibe el alumnado que fracasa o que termina abandonando.

\section{Método}

$\mathrm{El}$ acercamiento a la realidad educativa puede hacerse con procedimientos diversos en función de los propósitos marcados y en coherencia con los supuestos epistemológico del enfoque metodológico adoptado. Cuando lo que se busca es la comprensión desde la trama de significaciones y representaciones que construyen los sujetos sobre la realidad y que aportan las claves con las que interpretar esta, la opción metodológica es la cualitativa, y esta es la que ha sido asumida, dado que ese era nuestro objetivo. Asimismo, poner en valor las voces de los sujetos vinculados al fracaso y al abandono educativo, colocando sus narraciones en el centro de la investigación, es un modo de reconocerlos como agentes políticos capaces de hablar y reflexionar por sí mismos sobre sus situaciones y experiencias de fracaso y abandono. Al mismo tiempo, atender a las voces del alumnado es disponer de una valiosa herramienta para generar mejoras en los centros escolares y desarrollar prácticas docentes más justas e inclusivas (Sandoval, Echeita y Simón, 2015). Al fin y al cabo, son quienes se convierten en el punto de mira cuando se aborda esta problemática, si bien quedan como referentes implícitos (Gimeno Sacristán, 2003, p.17).

Tres han sido las estrategias de recogida de información: las entrevistas etnográficas semiestructuradas, estudios de casos biográficos y grupos focales, resultando un total de 91 (el 33\% mujeres y el 67\% hombres), 9 y 3, respectivamente. La recogida de información se ha realizado en Cádiz, Málaga y Almería (España), dado que era el contex to indagatorio contemplado. Los sujetos que han participado tienen edades comprendidas entre 12 y 26 años: el 46,3\%, entre 12 y 16 años (cursan ESO), y el 53,7\% entre 17 y 26 años (sujetos que realizan FPB, FP de Grado Medio y Grado Superior, PCPI, ESPA y estudios de Magisterio). Se aportan testimonios de adolescentes y jóvenes, cuyos datos de identificación son el género, la edad y los estudios que realizaban en el momento de la investigación, si es que lo hubieran retomado. Para el tratamiento de los datos se empleó el software Nvivo11. La gestión de la calidad de la investigación se ha garantizado mediante la triangulación (entre investigadores, fuentes de datos, con las personas 
participantes, con otras investigaciones). Igualmente, los principios éticos que han guiado este proceso indagatorio han sido, fundamentalmente, la negociación, la confidencialidad, la equidad, la participación y el compromiso con el conocimiento, siempre bajo el consentimiento informado de las familias (menores de edad) y los sujetos.

\section{La matriz de dominación del fracaso escolar y del abandono educativo}

El análisis de los datos nos ha permitido situar el fracaso escolar y el abandono educativo en la matriz de dominación que se configura a partir de la interconexión e interdependencia de sistemas, estructuras y dinámicas que provocan, de un modo u otro y con diferente alcance, situaciones de desigualdad y exclusión. Hill Collins (1990) la define como "la organización general de las relaciones jerárquicas de poder en cualquier sociedad” (p. 299), y en cualquier institución, incluida la educativa. Desde esta consideración, "cualquier matriz particular de dominación se organiza a través de cuatro dominios de poder interrelacionados: estructurales, disciplinarios, hegemónicos e interpersonales" (p. 276). Los cuatro dominios entran en juego en la conformación del fracaso escolar y abandono educativo, incidiendo en su desarrollo. Dicha matriz permite realizar un análisis situado del sujeto y de sus experiencias en relación al fracaso y/o al abandono.

a) El dominio estructural. Hace alusión a las estructuras que producen, mediante sus propios mecanismos, procesos que pueden generar o intensificar la desigualdad, la inequidad, la marginación y la injusticia, y entre las que cabe mencionar la economía, las regulaciones normativas, las agencias políticas (sociales, educativas, culturales, comunitarias, etc.), la organización del sistema educativo, la privatización de los servicios públicos, la austeridad presupuestaria, los recortes ${ }^{3}$, etc. Las estructuras y sus dispositivos de acción movilizan procesos que llegan a ser limitantes, no por igual para todos los grupos sociales y colectivos, sino especialmente para aquellos que el disfrute de oportunidades, no ya de partida sino de resultados, se ve mermado. Así, la precariedad laboral de los miembros de la unidad familiar, la presencia de situaciones de desempleo por parte de uno o de los dos progenitores, la escasez de recursos para atender las necesidades básicas, etc., conllevan el empobrecimiento de las unidades familiares con repercusiones a diferentes niveles (salud, relaciones, etc.).

su padre no trabajaba durante el mes de septiembre y no recibía un salario hasta octubre, y por esa razón, a principios del curso, aunque contaba con los libros de texto, no disponía de todo su material escolar. Llevaba a clase los lápices del año anterior, pero no bolígrafos (ya que en los cursos previos no los utilizaban y esas primeras semanas continuaba sin ellos) y esto motivó el enfado de la maestra, que le espetó: «Pues si no traes un boli rojo y uno azul ¿̇para qué vienes a clases?, ¡mejor no vengas a clase!». Dice que se lo tomó al pie de la letra y que a partir de ese momento cada semana tenía una excusa para no ir a clase: estaba enferma, le dolía la cabeza, la barriga. (Calvo García, 2018, p. 56)

Decían que yo era un niño bajuno (...) mi familia no es de dinero, mis padres no están trabajando ninguno y yo no puedo vestir como ellos así, yo me visto normal y corriente (...) yo consigo mi dinero y me lo puedo comprar, pero antes iba siempre con un

${ }^{3}$ Véase Save the Children (2019). 
chándal, siempre manchado con la ropa partía y todo, ya no* (Chico, 15 años, $2^{\circ}$ $\mathrm{ESO}$ )

[su situación] realmente mal porque no tengo para llevar pan a mi familia, para ayudar a mi familia, porque tengo dos hermanas más pequeñitas que están estudiando, hace falta el dinero para por lo menos un plato combinado o si le hacen falta a las niñas unos tenis pa'gimnasia, o un chándal para el colegio y... A mí me gustaría decirle a mi madre, toma mamá compra el chándal para el colegio o compra. $\Upsilon$ eso, que lo paso muy mal. A ver si encuentro algo también páechar una mano. (Chico, 19 años)

El riesgo de pobreza no determina por sí mismo el fracaso y el abandono educativo, pero sí podemos señalar que es un factor que interviene en su concurrencia (Bonal y Tarabini, 2010; Calderón, 2014; Leithwood, Harris y Strauss, 2010; Mooney, Knox y Schacht, 2011; Rogero Anaya, 2012); es una fuente de riesgo (at risk) para el sujeto porque hace que tenga más probabilidad de tener consecuencias adversas o desfavorables (Finn y Rock, 1997), como puede ser repetir, fracasar, abandonar. Como recoge Llano Ortiz (2019, p.15),

\begin{abstract}
ser un menor pobre es muy distinto de no serlo: el 24,8\% vive en hogares en los cuales se han producido uno o más retrasos en el pago de la hipoteca o alquiler del hogar; el $21 \%$ en hogares que no pueden mantener la vivienda a temperatura adecuada en invierno; el 27,1\% vive en hogares que tienen mucha dificultad para llegar a fin de mes y el 21,6\% de la población infantil vive en hogares que no pueden permitirse tener un ordenador. Las cifras de estos cinco indicadores quintuplican por lo menos las que se registran entre la población menor que no es pobre.
\end{abstract}

A ello se le añade también los modelos de apoyo que se adoptan para el alumnado que no sigue el ritmo de los demás, alumnado que en muchas ocasiones termina repitiendo curso, y en otras tantas abandonando el sistema prematuramente (Choi, 2017, p. 7), resultando ser una medida educativa poco efectiva, y "socialmente muy injusta [pues] el alumnado pobre repite cuatro veces más que el de más recursos" (Ferrer, 2019, p. 2)5; también la marginación política en términos de "exclusión en la democratización" (MartínezPalacios, 2017) o no participación como agente con voz propia (Finn y Rock, 1997; Jensen, 2013). Estas situaciones mostradas evidencian la incidencia este dominio en la producción de la opresión y las injusticias, y también en el desarrollo de estrategias de resistencia (Alonso, Anderson, Su y Theoharis, 2009; Willis, 1988) por el alumnado cuyas necesidades no reciben respuestas parte del sistema educativo como también de otros (económico, social, etc.). La pobreza y la exclusión, entre otras fuentes, deben ser estimadas por las políticas educativas para evitar que queden enmascaradas y se tomen el fracaso escolar y el abandono como efecto de problemas y déficits personales, de deficiencias familiares y de grupo concretos (étnico, cultural, etc.). Omitir la fuente y no analizar sus efectos a nivel educativo y social es una invitación directa a la reproducción de desigualdades e injusticias (sociales y educativas), que terminan culpando al alumnado y a sus familias por sus circunstancias vitales, y haciéndoles por ello responsables. Las situaciones de riesgo son el efecto de condiciones estructurales que generan dinámicas sociales, económicas, políticas y culturales más amplias, que inciden y se entrelazan con la educación y las prácticas docentes que se llevan a cabo en las instituciones escolares. Ignorar tales condiciones es contribuir al mantenimiento de estructuras y prácticas injustas,

\footnotetext{
${ }^{4}$ Se ha respetado las formas propias de expresión de las personas participantes.

${ }^{5}$ Murillo y Martínez-Garrido (2018, p. 52) concluyen que "España es uno de los países con el sistema educativo más segregado de toda la Unión Europea, y muy alejado de los países de su entorno más cercano”.
} 
discriminatorias y no equitativas (Portelli, Shields y Vibert, 2007; Vasudevan y Campano, 2009).

b) El dominio disciplinario. Sin quedar al margen del estructural, este dominio cumple la función de controlar, gestionar y organizar el comportamiento de los sujetos bajo el habitus institucional, el cual marca las experiencias, las expectativas, las decisiones, los ritmos, las relaciones, las trayectorias del alumnado (Diamond, Randolph y Spillane, 2004; Tarabini, Curran y Fontdevila, 2015), y tiene un efecto relevante que determina los procesos educativos. Este habitus hallará expresión desde su concreción en prácticas docentes distintas (Reay, David y Ball, 2001) con repercusión diferente según sea el estudiantado y su realidad, condicionando la dirección de cada cual. Toman presencia el orden, el control, la vigilancia y sus dispositivos de acción como pueden ser los partes de incidencias y las expulsiones, que más que medidas educativas son mecanismos de la tecnología disciplinaria inherente a la institución educativa; también formas verbales (como dar chillidos y gritar) y conductas con las que dejar constancia de la relación asimétrica y de que lo que hace el alumnado no responde a lo esperado conforme al habitus:

\begin{abstract}
To a lo mejor entraba en la clase y ponía el parte a las siete de la mañana, pegado en la puerta. No sé por qué (risas). To siempre me lo he preguntado... A lo mejor nos tocaba a primera (hora), y...nos mandaba para la sala de expulsados sin motivo alguno. Al acabar de llegar tú con los ojos pegados al instituto, y te ves el parte, digo «३̈en serio?». (Chico, 17 años, $\left.2^{\circ} \mathrm{ESO}\right)$

Por nada me ponía un parte, decía «para abajo» por nada que hacía, estornudaba, para abajo, hiciera lo que hiciera para abajo. (Chico de 15 años, PCPI)

$\Upsilon$ recuerdo que el profesor tenía muy mala leche y... y cogía y... y cuando no le gustaba algo que... lo que sea, la forma de escribir, porque él tenía la costumbre de que quería que escribiera todo seguido. To tenía 8 añitos. $\Upsilon$... y cogía y entre la mesa me daba patadas a la barriga. (Chico, 20 años, $2^{\circ}$ ESPA)

Por nada que haga, ya me está chillando, y chillar no veas cómo chilla, y así no ayuda [se refiere al jefe de estudios]. (Chico, 18 años, $4^{\circ} \mathrm{ESO}$ )

Nunca me ha gustado estudiar (...). To es que me veo... hasta aquí, ahora mismo, me siento agobiado en una silla sentado y... yo me agobio... no puedo estar seis horas a piñón fijo. Si fueran tres horas, vale...es que me agobio, me emparanoio. (Chico, 17 años, $\left.4^{\circ} \mathrm{ESO}\right)$
\end{abstract}

Este orden disciplinario sirve para mantener las relaciones jerárquicas encubiertas bajo el cumplimiento del currículum escolar al que se ha de adaptar el alumnado, y es esto lo que justifica que se tenga que preservar la posición que cada cual (profesorado y alumnado) tiene que ocupar en el marco de relaciones académicas. Las relaciones jerárquicas forman parte del currículum oculto, incidiendo en la conservación del habitus institucional, y los indicios se evidencian con la aplicación de, entre otros dispositivos, los partes y las expulsiones. Los mecanismos que se adoptan no ayudan, realmente, a que reflexionen sobre su comportamiento. No es ser permisivo, sino asumir procedimientos que garanticen una convivencia democrática y sin violencia. Las buenas intenciones que llevan adosadas los dispositivos se tornan en estrategias tanto para el profesorado como para el alumnado: para el primero, es un modo de no tener en el aula al alumnado, y para el segundo, el motivo para ser expulsado de un contexto del que quiere escapar:

Porque me canso de alli [instituto], cada dos por tres llamando a mi madre. Voy a lo mejor a una clase y me ponen falta sin que haya hecho nada, porque ya están acostumbrados a eso, a que falte. $Y$ han llamado muchas veces a mi madre diciendo mentiras. (Chico, 15 años, $2^{\circ} \mathrm{ESO}$ ) 
A mí me llamó mucho la atención, porque... muchas veces en los colegios, y esto sí que yo lo... lo valoré, hay gente que le echan de la clase o le expulsan y tal y cual, y lo único que quieren es quitárselo de encima. (Chico, 20 años, $2^{\circ} \mathrm{ESPA}$ )

Por su propio sentido y función, el dominio disciplinario no queda circunscrito al ámbito educativo. Este forma parte de una sociedad disciplinaria y de control que es también del rendimiento (Han, 2012). Utiliza procedimientos para justificar, científicamente, las categorías con las que clasificarlos, lo que merece cada grupo y lo que se espera de cada cual. Se busca la eficiencia al tiempo que con ello se refuerzan las injusticias (para algunos) y los privilegios (para otros). O’Neil (2018) es clara al respecto en el análisis que realiza sobre el poder de los algoritmos y sus consecuencias catastróficas para la igualdad de oportunidades y la justicia:

$$
\begin{aligned}
& \text { el resultado es un sistema educativo que favorece a los privilegiados. Es un sistema } \\
& \text { que actúa en contra de los estudiantes más necesitados, excluyendo a la gran mayoría } \\
& \text { de ellos (y empujándolos por un camino que conduce a la pobreza). (p. 83). }
\end{aligned}
$$

c) El dominio interpersonal. Apunta a las relaciones personales y las interacciones que forman parte del mundo experiencial y vital del alumnado, que se construyen y manifiestan en los diferentes contextos a los que pertenece, y en las que están implicados profesorado, compañeros, familiares, amistades, etc. Estas relaciones personales e interacciones no se producen al margen de las relaciones de poder del dominio disciplinario ni estructural. Parece que la dimensión humana de toda relación interpersonal queda determinada por lo académico y curricular, y a ello se debe. Las relaciones adoptan un carácter técnico-instrumental para poder cubrir las exigencias curriculares. Cumple una función reguladora y de control que no se dispensa por igual a todo el alumnado; se produce una marginación afectiva -injusticia de igual índole- que es la que experimenta el estudiantado marcado por el estigma de la repetición de curso, los suspensos, las expulsiones, los partes de incidencia. Se levanta un muro que imposibilita la escucha, el diálogo y la negociación, y provoca la puesta en marcha de procesos que invitan al desenganche de lo escolar por el alumnado, y en muchos casos, a la rendición. Como señala González González (2015), las relaciones personales y académicas inciden en la no-implicación del alumnado, y en ello se pone en juego en qué medida se "sienten recibidos, respetados, apoyados y escuchados" (p.167).

\footnotetext{
Me da cosa preguntarle y a veces lo pregunto y... no me hacen caso. (Chico, 16 años, $\left.3^{\circ} \mathrm{ESO}\right)$

No, no explicaban, y lo poco que explicaban no me enteraba tampoco (...) terminé por no preguntar porque tampoco me respondían. (Chico, 15 años, $2^{\circ} \mathrm{ESO}$ )

Sí, allí [instituto] era todo más... más frío, como si fuera uno más o nadie. (Chico, 23 años, $1^{\circ} \mathrm{ESPA}$ )

La profesora que tenía de apoyo se metía muchas veces conmigo, a lo mejor cuando hablábamos de un tema iba... "porque es que tú estás solo en el recreo, tal y cual...", pero nunca...no era ayuda, nunca había ayuda. (Chico, 20 años)

Yo era muy torpe para estudiar y todo, pero aparte tampoco los maestros decían si tú apruebas, sacas las tareas bien, luego en el examen te va a contar... no, después del examen na, yo llegaba al examen y suspendía decía, la asignatura suspendía ya, no le prestaba interés al instituto. (Chica, 24 años, $2^{\circ} \mathrm{ESPA}$ )
}

Las relaciones de las que es partícipe el estudiantado tienen incidencia en la construcción de su identidad, autoestima y autoconcepto: los maestros se creen que yo no sirvo para nada, soy la oveja descarrilada, soy un caso perdido, soy muy torpe, mi madre que ya me daba por perdida, yo soy muy bruta, era un trasto muchas veces, etc. Una construcción 
que no se produce al margen de la amalgama de factores que se cruzan en las vidas de los sujetos, y que se realiza bajo el amparo de escenarios sociales con los que dialogan. La identidad que estos jóvenes construyen depende, de modo crucial, de las relaciones dialógicas, de ahí la importancia que tiene el reconocimiento y las condiciones en las que se produce de forma exitosa (igualdad y justicia) o no (desigualdad e injusticia) (Taylor, 1996, 2009). Por tanto, las relaciones interpersonales, ante la ausencia de reconocimiento o falso reconocimiento, pueden llegar a ser un espacio para el ejercicio del poder, de la dominación, la opresión. De igual manera, las relaciones interpersonales con los iguales compañeros y compañeras de clase o amistades) y las familias también tienen su lugar en este dominio.

d) El dominio hegemónico. Este último, estrechamente interconectado con los anteriores, se vincula con el lenguaje empleado, los materiales curriculares (especialmente los libros de textos), la cultura oficial, los contenidos curriculares, las estrategias metodológicas, la evaluación (exámenes), los modelos de géneros, la organización de los espacios y tiempos, etc. Dominan los códigos elaborados frente a los restringidos, se antepone la cultura oficial y curricular a la vinculada a las experiencias y la vida cotidiana del alumnado, prevalece una relación disciplinar frente a otra basada en la escucha, se silencian más las voces de grupos o minorías, se aceptan menos los sujetos que se muestran rebeldes y disidentes.

\begin{abstract}
que me lo explicaran más fácil, no así, que ellos parecen médicos explicando y no me entero de nada y luego en el examen, a decir lo mismo. (Chico, 17 años)

lo que me pasa es eso, que vengo al colegio y estudio doce asignaturas, que de las doce me interesan a lo mejor tres, de doce, religión: no voy a ser católica, educación física: no voy a hacer deporte porque yo no voy a hacer deporte, yo qué sé, fisica y química: no voy a hacer nada relacionado con eso, tecnología: no voy a hacer nada... ¿ sabes lo que te quiero decir? Son asignaturas que en mí sobran, que en mi digo ¿para qué? (Chico, 14 años, $\left.2^{\circ} \mathrm{ESO}\right)$

como yo jugaba al fútbol, pues siempre me estaban diciendo que yo era un marimacho. (Chica, 25 años, $\left.2^{\circ} \mathrm{ESPA}\right)$

sí, me fui al PCPI ese y ahí yo básicamente ya no era agresivo, es que claro mi madre todos los días una pelea, y ahí ella que deja de pelearte, que pasó, la que me crio ella me metió eso que un día otro y otro y eso, porque pego, sí que estás solo y me fue metiendo todo eso. (Chico, 16 años, PCPI)

pasan de mí, totalmente vaya, como si no existiera ni me pasaran cosas, cosas además importantes. (Chica, 18 años, PCPI)

mi padre era más gitano, entonces como que era más... un poquito más discriminada. (Chica, 19 años, $2^{\circ}$ ESPA)
\end{abstract}

\title{
5. Conclusiones
}

Asumir la herramienta de la interseccionalidad es luchar por los derechos de quienes son excluidos, discriminados, marginados desde un proyecto social y político comprometido con el bien común, la equidad y la justicia social. Nos permite hacer análisis con los que liberar a los sujetos de la culpa, porque la ideología neoliberal bien se encarga de generar en los sujetos el sentimiento de que sus problemas se deben a ellos mismos, son sus responsables y, por ende, son quienes tienen que buscar la solución. "El neoliberalismo niega unas condiciones previas que conducen a desigualdades estructurales; en consecuencia, se complace por desmantelar las políticas y desacreditar los movimientos 
preocupados por las estructuras que generan injusticia” (Bilge, 2013, p. 407), sin olvidar que tales políticas se han apropiado de la inclusión, la justicia y la equidad en aras de una clara estandarización basada en el éxito y la excelencia (Portelli, Shields y Vibert, 2007).

Desde el análisis realizado, que ha permitido configurar la matriz de dominación del fracaso escolar y del abandono educativo, es posible apreciar la complejidad de tales fenómenos, no reducibles al sujeto. Incorporar la herramienta de la interseccionalidad para el análisis de los mismos, nos permite reforzar la idea de que no son neutrales, estáticos e inmanentes. Ambos se van configurando y tomando presencia desde la interseccionalidad que se produce entre los diferentes sistemas con los que el alumnado mantiene, de un modo u otro, un vínculo; sistemas que atraviesan sus experiencias y las determinan. Los cuatro dominios, identificados desde su particularidad, no se manifiestan de modo independiente, sino de manera interrelacionada, a modo de maraña (Platero, 2014).

Esta configuración de la matriz de dominación no se manifiesta en igual medida para todos los posibles casos de fracaso y abandono. Recordando que la interseccionalidad pone en valor las experiencias de los sujetos para poder comprender la expresión de tales fenómenos, y nosotros las hemos tomado a través de los testimonios de adolescentes y jóvenes, es necesario considerar los aspectos comunes que el estudiantado que pasa a formar parte de la categoría fracaso o abandono puede compartir. Tales aspectos son una vía para poder constatar que en todos inciden los sistemas de desigualdad, en cada estudiante, dichos sistemas varían; consecuentemente, sus efectos son diferentes en grado y presencia. La interseccionalidad entre sistemas existe con una concreción distinta según cada sujeto en sus contextos particulares, y es la que ayuda a entender las experiencias y el lugar social del sujeto en clave de entrelazamiento de sistemas de dominación (desigualdad). También ayuda a visibilizar cómo influyen en el reconocimiento que reciben los sujetos y en lo que se espera de ellos. Ayuda, por tanto, a comprender el fracaso escolar y el abandono educativo desde la multiplicidad y variabilidad de realidades humanas.

$\mathrm{El}$ análisis interseccional evita que el alumnado segregado se vea excluido dentro de las propuestas que se diseñan para garantizar la igualdad y la inclusión educativa. Cuando se toma al alumnado que abandona como una categoría estandarizada, eliminando al sujeto y a sus experiencias del análisis, implica seguir contribuyendo a mantener su exclusión y segregación, en la medida en que no se toman en consideración los diferentes tipos de desigualdades que experimenta más allá de lo que es parte de su experiencia propia. No podemos desvincular, por tanto, el análisis del fracaso y del abandono del análisis de factores estructurales, porque la consecuencia que se deriva es reducir el análisis de tales fenómenos a cuestiones individuales. Se individualiza la problemática y se autoresponsabiliza al alumnado (causas individuales-respuestas individuales), propio del neoliberalismo progresista (Fraser, 2018).

Finalmente, es necesario abandonar los discursos del déficit, los centrados exclusivamente en las condiciones individuales ignorando las condiciones y los contextos social, económico y político, los discursos educativos oficiales basados en una visión instrumental de lo que significa ser estudiante para pasar a una educación crítica que ofrece nuevas formas de pensar y construir el currículum y la enseñanza, al tiempo que reconoce "explícitamente la naturaleza política de la escolarización y el papel del poder y los privilegios en el mantenimiento de estructuras y prácticas no equitativas" (Portelli, Shields y Vibert, 2007, p. 14). El encuentro entre la interseccionalidad y la educación crítica es claramente posible (Hill Collins y Bilge, 2019), y en él debemos confiar para 
actuar. Este es el camino para hacer que la educación sea liberadora, problematizadora y constituyente de un sujeto con reconocimiento humano, político y social. La educación es un derecho humano, y como tal, debe ser respetuosa y tolerante con el conjunto de posibilidades humanas que representan los estudiantes, rechazando lo intolerable, aquello que atente contra los derechos humanos e impida la equidad.

\section{Agradecimientos}

La investigación se desarrolló bajo el proyecto "Riesgo de fracaso y abandono escolar en Secundaria Obligatoria. Los contextos educativos, familiares y socioculturales. Un estudio cualitativo" (SEJ-2664), Consejería de Innovación, Ciencia y Empresa, Junta de Andalucía (2014-2018).

\section{Referencias}

Alonso, G., Anderson, N. S., Su, C. y Theoharis, J. (2009). Our schools suck students talk back to a segregated nation on the failures of urban education. University Press.

Bernardi, F. y Requena, M. (2010). Inequality in educational transitions: The case of postcompulsory education in Spain. Revista de Educación, núm. extraordinario, 93-118.

Breen, R. y Jonsson, J. O. (2005). Inequality of opportunity in comparative perspective: Recent research on educational attainment and social mobility. Annual Review of Sociology, 31, 223243. https://doi.org/10.1146/annurev.soc.31.041304.122232

Bilge, S. (2013). Intersectionality undone. saving intersectionality from feminist intersectionality studies. Du Bois Review: Social Science Research on Race, 10(2), 405-424. https://doi.org/10.1017/S1742058X13000283

Bonal, X. y Tarabini, A. (2010). Ser pobre en la escuela. Habitus de pobreza y condiciones de educabilidad. Miño y Dávila.

Calderón, I. (2014). Sin suerte, pero guerrero hasta la muerte: Pobreza y fracaso escolar en una historia de vida. Revista de Educación, 363, 184-209. https://doi.org/10.4438/1988-592X-RE-2012-363-177

Calderón, I. (2016a). Fracaso escolar y desventaja sociocultural. Una aproximación biográfica. UOC.

Calderón, I. (2016b). Liberarse de la escuela. Historia de vida de Elena. RIUMA.

Calero, J. (2007). Desigualdades socioeconómicas en el sistema educativo español. Ministerio de Educación y Ciencia.

Calero, J., Choi, A. y Waisgrais, S. (2010). Determinantes del riesgo de fracaso escolar en España: Una aproximación a través de un análisis logístico multinivel aplicado a PISA-2006. Revista de Educación, núm. extraordinario, 225-256.

Calvo García, G. (2018). Marta nos habla: Me voy a sacar mis estudios y voy a ser lo que yo quiera. En R. Vázquez (Coord.), Hacia una literacidad del fracaso escolar y del abandono temprano desde las voces de adolescentes y jóvenes (pp. 53-74). Editorial UCA.

Carabaña, J. (2009). Una vindicación de las escuelas españolas. FRC: Revista de Debat Polític, 21, 6883.

Comisión Europea. (2010). Europa 2020: Una estrategia para el crecimiento inteligente, sostenible e integrador. Publicaciones UE. 
Crenshaw, K. (1991). Mapping the margins: Interseccionality, identity politics and violence againts women of color. Stanford Law Review, 43(6), 1241-1299.

https://doi.org/10.2307/1229039.

Choi, A. (2017). Efectos de la repetición de curso y alternativas. Fundación Alternativas.

Diamond, J. B., Randolph, A. y Spillane, J. P. (2004). Teachers' expectations and sense of responsibility for student learning: The importance of race, class, and organizational habitus. Anthropology \& Education Quarterly, 35(1), 75-98.

https://doi.org/10.1525/aeq.2004.35.1.75

Entorf, H. y Minoiu, N. (2005). What a difference immigration policy makes: A comparison of PISA scores in Europe and traditional countries of immigration. German Economic Review, 6(3), 355-376. https://doi.org/10.1111/j.1468-0475.2005.00137.x

Escudero, J. M. (2005). Fracaso escolar, exclusión educativa: ¿De qué se excluye y cómo? Profesorado. Revista de Currículum y Formación del Profesorado, 1(1), 1-23.

Escudero, J. M. y Martínez, B. (2012). Las políticas de lucha contra el fracaso escolar: ¿Programas especiales o cambios profundos cambios profundos del sistema y la educación? Revista de Educación, núm. extraordinario, 174-193.

Escudero, J. M., González, M. T. y Martínez, B. (2009). El fracaso escolar como exclusión educativa: comprensión, políticas y prácticas. Revista Iberoamericana de Educación, 50, 41-64

Fallis, R. K. y Opotow, S. (2003). Are students failing school or are schools failing students? Class cutting in high school. Social Issues, 59(1), 103-119. https://doi.org/10.1111/15404560.00007

Ferguson, B., Tilleczek, K., Boydell, K. y Rummens, J. A. (2005). Early school leavers: Understanding the lived reality of student disengagement from secondary school. Ontario Ministry of Education and Training.

Fernández, M., Mena, L. y Riviére, J. (2010). Fracaso y abandono escolar en España. Fundación La Caixa.

Ferrer, Á. (2019). Todo lo que debes saber de PISA 2018 sobre equidad. Save the Children.

Ferrer, G., Castel, J. L. y Ferrer, F. (2006). Las desigualdades del sistema educativo a través del estudio PISA 2003. Revista de Educación, núm. extraordinario, 399-428.

Finn, J. D. y Rock, D. A. (1997). Academic success among students at risk for school failure. Journal of Applied Psychology, 82(2), 22 1-234. https://doi.org/10.1037/002 1-9010.82.2.221

Fraser, N. (21 de julio de 2018). ¿Podemos entender el populismo sin llamarlo fascista? Sin permiso. http://www.sinpermiso.info/textos/podemos-entender-el-populismo-sin-llamarlofascista-entrevista

Giddens, A. (2000). La tercera vía y sus críticos. Taurus.

Gimeno Sacristán, J. (2003). El alumno como invención. Morata.

González González, M. T. (2015). Los centros escolares y su contribución a paliar el desenganche y abandono escolar. Profesorado. Revista de Currículum y Formación del Profesorado, 19(3), 158 176. https://recyt.fecyt.es/index.php/profesorado/article/view/43639/25546

Han, B. (2012). La sociedad del cansancio. Herder.

Hill Collins, P. (1990). Black feminist thought. knowledge, consciousness and the politics of empowerment. Routledge. 
Hill Collins, P. (2009). Another kind of public education. Race, schools, the media and democratic possibilities. Beacon.

Hill Collins, P. y Bilge, S. (2019). Interseccionalidad. Morata.

Jensen, E. (2013). How poverty affects classroom engagement. Faces of Poverty, $70(8), 24-30$.

La Barbera, M. C. (2016). Interseccionalidad, un concepto viajero: Orígenes, desarrollo e implementación en la Unión Europea. Interdisciplina, 4(8), 105-122. https://doi.org/10.22201/ceiich.24485705e.2016.8.54971

Leithwood, K., Harris, A. y Strauss, T. (2010). Leading school turnaround: How successful leaders transform low performing schools. Jossey-Bass.

Llano Ortiz, J. C. (2019). El estado de la pobreza. Seguimiento del indicador de pobreza y exclusión social en España, 2008-2018. EAPN.

Marchesi, A. (2000). Un sistema de indicadores de desigualdad educativa. Revista Iberoamericana de Educación, 23, 135-163. https://doi.org/10.35362/rie2301009

Marchesi, A. (2003). El fracaso escolar en España. Fundación Alternativas.

McCall, L. (2005). The complexity of intersectionality. Signs, 30(3), 1771-1800. https://doi.org/10.1086/426800

Martínez-Palacios, J. (2017). Exclusión, profundización democrática e interseccionalidad. Investigaciones Feministas, 8(1), 53-71. https://doi.org/10.5209/INFE.54827

Mena, L., Fernández, M. y Riviére, J. (2010). Desenganchados de la educación: Procesos, experiencias, motivaciones y estrategias del abandono y del fracaso escolar. Revista de Educación, núm. extraordinario, 119-145.

Ministerio de Educación y Formación Profesional. (2020). La tasa de abandono escolar en España alcanza su nivel más bajo desde que se tienen datos. Ministerio de Educación y Formación Profesional.

Mooney, L., Knox, C. y Schacht, C. (2011). Understanding social problems. Wadsworth.

Mora, A. J. (2010). Determinantes del abandono escolar en Cataluña, más allá del nivel socioeconómico de las familias. Revista de Educación, núm. extraordinario, 171-190.

Moriña Díez, A. (2007). La exclusión social: Análisis y propuestas para su prevención. Fundación Alternativas.

Murillo, F. J. y Martínez-Garrido, C. (2018). Magnitud de la segregación escolar por nivel socioeconómico en España y sus Comunidades Autónomas y comparación con los países de la Unión Europea. RASE. Revista de Sociología de la Educación, 11(1), 37-58. https://doi.org/10.7203/RASE.11.1.10129

O’Neil, C. (218). Armas de destrucción matemática. Cómo el big data aumenta la desigualdad y amenaza la democracia. Capitán Swing.

Parrilla, A., Gallego Vega, C. y Moriña Díez, A. (2010). El complicado tránsito a la vida activa de jóvenes en riesgo de exclusión: Una perspectiva biográfica. Revista de Educación, 351, 211 233.

Platero, R. (2014). Metáforas y articulaciones para una pedagogía crítica sobre la interseccionalidad. Quaderns de Psicología, 16(1), 55-72. https://doi.org/10.5565/rev/qpsicologia.1219

Portelli, J. P., Shields, C. M. y Vibert, C.B. (2007). Toward an equitable education: Poverty, diversity and students at-risk. OISE/UT. 
Reay, D., David, M. y Ball, St. (2001). Making a difference? Institutional habit uses and higher education choice. Sociological Research Online, 5(4), 126-142. https://doi.org/10.5153/sro.548

Rogero, J. (2012). El fracaso escolar, causas y alternativas. En la Calle: Revista sobre Situaciones de Riesgo Social, 21, 9-13.

Román, M. (2013). Factores asociados al abandono y la deserción escolar en América Latina: Una mirada de conjunto. REICE. Revista Iberoamericana sobre Calidad, Eficacia y Cambio en Educación, 11(2), 23-37.

Romero, E. y Hernández, M. (2019) Análisis de las causas endógenas y exógenas del abandono escolar temprano: Una investigación cualitativa. Educación XX1, 22(1), 263-293.

Rujas, J. (2017). La construcción del fracaso escolar en España. Génesis y cristalización de un problema social. Papers, 102(3), 477-507. https://doi.org/10.5565/rev/papers.2297

Rumberger, R. W. (2001). Why students drop out of school and what can be done. Civil Rights Project.

Rumberger, R. W. (2011). Dropping out. Why students drop out of high school and what can be done about it. Harvard University Press.

Rumberger, R. W. y Ah Lin, S. (2008). Why students drop out of school: A review of 25 years of research. University of California.

Sandoval, M., Echeita, G. y Simón, C. (2015). Las voces de los estudiantes sobre la justicia y la equidad: Una experiencia en secundaria. Pulso, 39, 243-254.

Save the Children. (2019). Pobreza infantil. La percepción social de un problema invisible. Save the Children.

Susinos, T. y Calvo, A. (2005-2006). Yo no valgo para estudiar.... Un análisis crítico de la narración de las experiencias de exclusión social. Contextos Educativos: Revista de Educación, 9, 87-108. https://doi.org/10.18172/con.559

Tarabini, A. y Bonal, X. (2016). Los principios de un sistema educativo que no deje a nadie atrás. Save the Children.

Tarabini, A., Curran, M. y Fontdevila, C. (2015). El habitus institucional: Una herramienta teórica y metodológica para el estudio de la cultura escolar. Tempora. Revista de Sociología de la Educación, 18, 37-58.

Taylor, C. (1996). Fuentes del yo. La construcción de la identidad moderna. Paidos.

Taylor, C. (2009). El multiculturalismo y la política del reconocimiento. FCE.

Tilly, C. (2000). La desigualdad persistente. Manantial.

UNESCO. (2012). International standard classification of education. ISCED 2011. UNESCO-UIS.

Vasudevan, L. y Campano, G. (2009). The social production of adolescent risk and the promise of adolescent literacies. Review of Research in Education, 33(1), 310-353. https://doi.org/10.3102/0091732X08330003

Vázquez, R. (2018). Hacia una literacidad del fracaso escolar y del abandono temprano desde las voces de adolescentes y jóvenes: Resistencias, cicatrices y destinos. Editorial UCA.

Williamson, B. (1 de febrero de 2019). Education for the robot economy. Code Acts in Education. https://codeactsineducation.wordpress.com/2019/02/01/education-for-the-roboteconomy/

Willis, P. (1988). Aprendiendo a trabajar: Cómo los chicos de clase obrera consiguen trabajos de clase obrera. Akal. 
Witte, K., De Cabus, S., Thyssen, G., Groot, W. y Brink, H. M. (2013). A critical review of the literature on school dropout. Educational Research Review, 10, 13-28.

https://doi.org/10.1016/j.edurev.2013.05.002

\section{Breve CV de la autora}

\section{Rosa Vázquez}

Profesora Titular del Departamento de Didáctica de la Universidad de Cádiz. Ha participado en diversos proyectos de investigación (autonómico, nacional e internacional). Sus líneas de investigación están centradas en ámbitos diversos (escuelas rurales, dirección escolar, género) que tienen como elementos comunes la desigualdad, la inclusión y la justicia. Docente en estudios de grado, másteres y doctorado. Cuenta con publicaciones en revistas nacionales e internaciones y en editorial como Morata, Aljibe, Octadro, Miño y Dávila, Wolter Kluwer. Coordinadora del Grupo de Investigación L.A.C.E. (HUM 109) y Coordinadora del Programa de Doctorado Investigación y Práctica Educativa (8219) de la Universidad de Cádiz. ORCID ID: https://orcid.org/0000-00016595-177X. Email: rmaria.vazquez@uca.es 